\title{
DESIGN AND IMPLEMENTATION OF VARIABLE RADIUS SPHERE DECODING ALGORITHM
}

\author{
Wu Di, Li Dezhi and Wang Zhenyong \\ School of Electronics and Information Engineering, \\ Harbin Institute of Technology, Harbin, China \\ wudi45_2012@163.com, lidezhi@hit.edu.cn, ZYWang@hit.edu.cn
}

\begin{abstract}
Sphere Decoding (SD) algorithm is an implement decoding algorithm based on Zero Forcing $(Z F)$ algorithm in the real number field. The classical SD algorithm is famous for its outstanding Bit Error Rate (BER) performance and decoding strategy. The algorithm gets its maximum likelihood solution by recursive shrinking the searching radius gradually. However, it is too complicated to use the method of shrinking the searching radius in ground communication system. This paper proposed a Variable Radius Sphere Decoding (VR-SD) algorithm based on ZF algorithm in order to simplify the complex searching steps. We prove the advantages of VR-SD algorithm by analyzing from the derivation of mathematical formulas and the simulation of the BER performance between $S D$ and VR-SD algorithm.
\end{abstract}

\section{KEYWORDS}

MIMO, Signal Detecting, Maximum Likelihood Solution, Sphere Decoding, Variable Radius

\section{INTRODUCTION}

As the communication demand growing constantly, researchers have a stricter requirement for transform rate and BER performance of signal detection. Maximum Likelihood (ML) algorithm has the best BER performance among the MIMO signal detecting algorithms. The ML algorithm searches the feasible signal location in all possible areas, so it is seldom used in practical project. SD algorithm is an optimization algorithm of ML algorithm. The main strategy of SD algorithm is searching the feasible signal location within the super-sphere volume, when the radius is $R$. After finished one circulation, change the numerical value of $R$ and do the same thing with the new super-sphere, whose radius is new $R$. Finally find the signal located nearer than any other signals. The classical SD algorithm has two categories. One is called the SD algorithm based on breadth first search, the other is based on depth first search. The results of both search algorithms make no differences.

The main deficiency of classical SD algorithm is that the complexity is related to the original value of the convergence radius. If the original value of $R$ is too small, there will be no signal location in the super-sphere area, and the system will have to re-choose the original value of $R$ again. If we try to increase $R$ in some algorithms, it is necessary to take the step size into consideration. If the original value of $\mathrm{R}$ is too large, the system will take all the possible signal location into consideration, which makes the algorithm complexity comparable with ML's. In Jan Zizka et al. (Eds) : CCSEIT, MoWiN, IT, AIAP, ICBB - 2015 
addition, if the convergence algorithm of $R$ does not suit the situation, the computational complexity will make no difference with ML's. In that way, the SD algorithm may lose its significance of existence. In conclusion, whether the original value of the convergence radius is too large or too small, it will waste a lot of operands before it gets a suitable radius.

Considering all the analysis above, it demonstrates that the original value of the convergence radius has a great influence on the performance of classical SD algorithm. An excellent convergence strategy can greatly decrease the computational complexity of classical SD algorithm. An algorithm is proposed in this paper to calculate the original value of convergence radius to avoid a too large or small original value of the convergence radius. Besides, this paper proposed the VR-SD algorithm to find the target signal location after calculating the original convergence radius.

\section{AN IMPROVED STRATEGY OF SD ALGORITHM}

In this paper, the Multiple Input Multiple Output (MIMO) system deploys $M$ antennas to transmit the signal, and $N$ antennas to receive the signal $(M<N)$. Firstly, preprocess the channel state information (CSI) matrix $H$ to a square matrix. If $M=N$, the CSI matrix $H$ will be a square matrix without pre-processing. This paper only discuss the $M=N$ system.

In the assumption, the CSI matrix $\mathrm{H}$ can be transformed into real number by the functions below:

$$
\bar{H}=\left[\begin{array}{l}
\operatorname{Re}(H),-\operatorname{Im}(H) \\
\operatorname{Im}(H), \operatorname{Re}(H)
\end{array}\right]
$$

The size of $\bar{H}$ is $2 N \times 2 N$.

If $X$ and $Y$ are respectively transmit and receive matrix in complex number field, the transmit and receive matrix in real number field can be transformed in the following functions:

$$
\bar{X}=\left[\begin{array}{l}
\operatorname{Re}(X) \\
\operatorname{Im}(X)
\end{array}\right] \quad \bar{Y}=\left[\begin{array}{l}
\operatorname{Re}(Y) \\
\operatorname{Im}(Y)
\end{array}\right]
$$

If $\mathrm{Z}$ is the noise matrix, and $\bar{Z}$ is the noise matrix in real number field, the equation $\bar{Y}=\bar{H} \bar{X}+\bar{Z}$ still exists. The target of SD algorithm is to find the maximum likelihood solution in the convergence sphere, which is expressed as follows.

$$
\hat{x}=\underset{\bar{x} \in \Lambda}{\arg \min }\|\bar{y}-\bar{H} \bar{x}\|^{2}=(\bar{x}-\hat{\bar{x}})^{T} \bar{H}^{T} \bar{H}(\bar{x}-\hat{\bar{x}}) \leq R_{S D}^{2}
$$

$R_{S D}$ is the convergence radius. Let the center of the super-sphere be $\hat{\bar{x}}$ :

$$
\hat{\bar{x}}=\left(\bar{H}^{H} \bar{H}\right)^{-1} \bar{H}^{H} \bar{y}
$$


QR decomposes the matrix $\bar{H}$, and the $R$ matrix is an upper triangular matrix in real number field. The equation can be transformed as following:

$$
\begin{aligned}
(\bar{x}-\hat{\bar{x}})^{T} \bar{H}^{T} \bar{H}(\bar{x}-\hat{\bar{x}}) & =\|R(\bar{x}-\hat{\bar{x}})\|^{2} \\
& =\left\|\left[\begin{array}{ccccc}
r_{1,1} & r_{1,2} & r_{1,3} & \mathrm{~L} & r_{1,2 N} \\
0 & \mathrm{O} & \mathrm{L} & \mathrm{L} & r_{2,2 N} \\
0 & 0 & \mathrm{O} & \mathrm{L} & r_{3,2 N} \\
0 & 0 & 0 & \mathrm{O} & \mathrm{M} \\
0 & 0 & 0 & 0 & r_{2 N, 2 N}
\end{array}\right]\left[\begin{array}{c}
\bar{x}_{1}-\hat{\bar{x}}_{1} \\
\bar{x}_{2}-\hat{\bar{x}}_{2} \\
\bar{x}_{3}-\hat{\bar{x}}_{3} \\
\mathrm{M} \\
\bar{x}_{2 N}-\hat{\bar{x}}_{2 N}
\end{array}\right]\right\| \\
& =\left|r_{2 N, 2 N}\left(\bar{x}_{2 N}-\hat{\bar{x}}_{2 N}\right)\right|^{2}+\left|r_{2 N-1,2 N-1}\left(\bar{x}_{2 N-1}-\hat{\bar{x}}_{2 N-1}\right)+r_{2 N-1,2 N}\left(\bar{x}_{2 N}-\hat{\bar{x}}_{2 N}\right)\right|^{2}+\mathrm{L} \leq R_{S D}^{2}
\end{aligned}
$$

In the functions below, $R_{S D 0}$ stands for the original radius of convergence. In VR-SD algorithm, $R_{S D 0}$ is not set by empirical value but a serious of calculation. The function of $f\left(\bar{x}_{2 N}, \bar{x}_{2 N-1}, \mathrm{~L}, \bar{x}_{1}\right)$ is set to be:

$$
f\left(\bar{x}_{2 N}, \bar{x}_{2 N-1}, \mathrm{~L}, \bar{x}_{1}\right)=\left|r_{2 N, 2 N}\left(\bar{x}_{2 N}-\hat{\bar{x}}_{2 N}\right)\right|^{2}+\left|r_{2 N-1,2 N-1}\left(\bar{x}_{2 N-1}-\hat{\bar{x}}_{2 N-1}\right)+r_{2 N-1,2 N}\left(\bar{x}_{2 N}-\hat{\bar{x}}_{2 N}\right)\right|^{2}+\mathrm{L}
$$

If $\tilde{x}_{i}=\bar{x}_{i}-\hat{\bar{x}}_{i}$, the function of $f\left(\bar{x}_{2 N}, \bar{x}_{2 N-1}, \cdots, \bar{x}_{1}\right)$ can be transformed into:

$$
\tilde{f}\left(\tilde{x}_{2 N}, \tilde{x}_{2 N-1}, \cdots, \tilde{x}_{1}\right)=\left|r_{2 N, 2 N} \cdot \tilde{x}_{2 N}\right|^{2}+\left|r_{2 N-1,2 N-1} \cdot \tilde{x}_{2 N-1}+r_{2 N-1,2 N} \cdot \tilde{x}_{2 N}\right|^{2}+\cdots
$$

The original radius of convergence is chosen as $R_{S D 0}^{2}=\tilde{f}\left[\min \left(\tilde{x}_{2 N}, \tilde{x}_{2 N-1}, \cdots, \tilde{x}_{1}\right)\right]$. There are some parameters of matrix $R$ mixed in the function $\mathrm{f}$, so the value of $\mathrm{f}$ has little chance to be the minimum value or the maximum value on condition that each $\tilde{x}_{i}$ is in their own minimum value. In other words, the value of $\tilde{f}\left[\min \left(\tilde{x}_{2 N}, \tilde{x}_{2 N-1}, \cdots, \tilde{x}_{1}\right)\right]$ varies from the maximum value to the minimum value. In that case, the original value of the convergence radius cannot be set larger than the maximum value of $\tilde{f}\left[\min \left(\tilde{x}_{2 N}, \tilde{x}_{2 N-1}, \cdots, \tilde{x}_{1}\right)\right]$, or smaller than the minimum value, which can avoid many unnecessary calculations.

The variable radius algorithm will be used after $R_{S D 0}$ is calculated. The searching steps start from the bottom After calculating $\tilde{f}\left(\tilde{x}_{2 N}, 0, \cdots, 0\right)$ in different $\tilde{x}_{2 N}$, compare the results with $R_{\mathrm{s} \infty}^{2}$. Record all the possible values of $\tilde{x}_{2 N}$ which satisfied the inequation $\tilde{f}\left(\tilde{x}_{2 N}, 0, \cdots, 0\right) \leq R_{S D 0}^{2}$. After that, choose one random value of $\tilde{x}_{2 N}$ from its all possible values. Then calculate the value $\tilde{f}\left(\tilde{x}_{2 N}, \tilde{x}_{2 N-1}, 0, \cdots, 0\right)$ in different $\tilde{x}_{2 N-1}$ based on every possible $\tilde{x}_{2 N}$, and then compare the values between $\tilde{f}\left(\tilde{x}_{2 N}, \tilde{x}_{2 N-1}, 0, \cdots, 0\right)$ and $R_{S D 0}^{2}$. Record every combination of $\tilde{x}_{2 N}$ and $\tilde{x}_{2 N-1}$ which satisfied the inequation $\tilde{f}\left(\tilde{x}_{2 N}, \tilde{x}_{2 N-1}, 0, \cdots, 0\right) \leq R_{S D O}^{2}$. Iterate this algorithm until no $\tilde{x}_{i}$ can be found to satisfy the inequation $\tilde{f}\left(\tilde{x}_{2 N}, \tilde{x}_{2 N-1}, \cdots, \tilde{x}_{i}, \cdots, 0\right) \leq R_{S D D}^{2}$. Then step back to the upper layer. When the inequation $\tilde{f}\left(\tilde{x}_{2 N}, \tilde{x}_{2 N-1}, \cdots, \tilde{x}_{i+1}, \cdots, 0\right) \leq R_{S D 0}^{2}$ is satisfied, change the value of $\tilde{x}_{i+1}$, and restart the comparison in layer $i$ again. If an $i$ satisfies the inequation $\tilde{f}\left(\tilde{x}_{2 N}, \tilde{x}_{2 N-1}, \cdots, \tilde{x}_{i}, \cdots, 0\right) \leq R_{S D O}^{2}$, it means the original convergence radius $R_{s D 0}$ needs to be increased so that the inequation can establish. On the contrary, if after one circulation, there are more than one group of $\left[\tilde{x}_{2 N}, \tilde{x}_{2 N-1}, \cdots, \tilde{x}_{1}\right]$ satisfy the inequation $\tilde{f}\left(\tilde{x}_{2 N}, \tilde{x}_{2 N-1}, \cdots, \tilde{x}_{1}\right) \leq R_{S D}^{2}$, it means 
the $R_{s D 0}$ need to be decreased. After decreasing the $R_{s D 0}$ to $R_{s D}$, repeat the comparison circulation till there only exists one group of $\left[\tilde{x}_{2 N}, \tilde{x}_{2 N-1}, \cdots, \tilde{x}_{1}\right]$ that make the inequation $\tilde{f}\left(\tilde{x}_{2 N}, \tilde{x}_{2 N-1}, \cdots, \tilde{x}_{1}\right) \leq R_{S D}^{2}$ established. Then the solution, $\left[\tilde{x}_{2 N}, \tilde{x}_{2 N-1}, \cdots, \tilde{x}_{1}\right]$, can transform into the real number signal matrix $\hat{\bar{x}}=\left[\bar{x}_{2 N}, \bar{x}_{2 N-1}, \cdots, \bar{x}_{1}\right]$, and the target signal in complex number area can be expressed as :

$$
\hat{x}=\left[\bar{x}_{2 N}+j \bar{x}_{N}, \bar{x}_{2 N-1}+j \bar{x}_{N-1}, \cdots, \bar{x}_{N}+j \bar{x}_{1}\right]_{1 \times N}
$$

\section{THE EXPERIMENTS OF VR-SD ALGORITHM}

This paper compares the performance between classical SD algorithm and VR-SD algorithm in $2 \times 2$ MIMO system using QPSK modulation. Knowing from the aforementioned analysis, if the convergence circulation is free from loop times, the performance of classical SD algorithm will be the same as ML algorithm's. The circulation of classical SD algorithm may not keep doing until it finds the final target signal, because the CSI keeps changing during the process of implementation. If the time overhead of signal detection algorithm is too large, its CSI may change before it finishes the calculation. Furthermore, since the signal is being transferred all the time, if the system wastes too much time in signal detection, the subsequent signal will jam together. Considering all the situation above, the times of convergence circulation of classical SD algorithm will be constant during the simulation in this paper. After the times of convergence circulation reach the setting number, the system will choose a signal in the super-sphere of the last circulation as the target signal randomly, and force the algorithm to exit the circulation.

To get the convergence time of different convergence circulations of classical SD algorithm, this paper did the simulation of classical SD algorithm within the limited convergence times of 4, 5, 6 and 7. On the condition of the same hardware and the same version of MATLAB software, compare the convergence time between the limited times classical SD algorithm and the unknown convergence times VR-SD algorithm. The simulation is done in $2 \times 2$ MIMO system using QPSK modulation with the $2^{20}$ code length. As the variance of each kind of simulation is small, we do each kind of simulation ten times and calculate the average time. The results are shown in table 1:

Table 1. Calculation time of VR-SD and classical SD

\begin{tabular}{|l|l|l|}
\hline Algorithm & Calculation time (s) & Convergence times \\
\hline VR-SD & 136.2 & N/A \\
\hline \multirow{4}{*}{ Classical SD } & 120.7 & 4 \\
\cline { 2 - 3 } & 142.1 & 5 \\
\cline { 2 - 3 } & 148.5 & 6 \\
\cline { 2 - 3 } & 150.3 & 7 \\
\hline
\end{tabular}

By comparing the convergence time of classical SD algorithm, it is concluded that the calculation time rises with the rising convergence times. The calculating time difference between 4 and 5 times is nearly 22 seconds, but the time difference between 5 and 6 times sharply decreases to just over 6 seconds. The time difference between 6 and 7 times is even less. This shows that with the increasing convergence times, the percent of classical SD algorithm finishes their calculation before reaching the limit convergence times is increasing. From Table 1, the calculating time of VR-SD algorithm is close to the classical SD algorithm when the convergence times is 5 . In the following simulation, we compare the BER between VR-SD and the classical SD algorithm with 5 convergence times. 


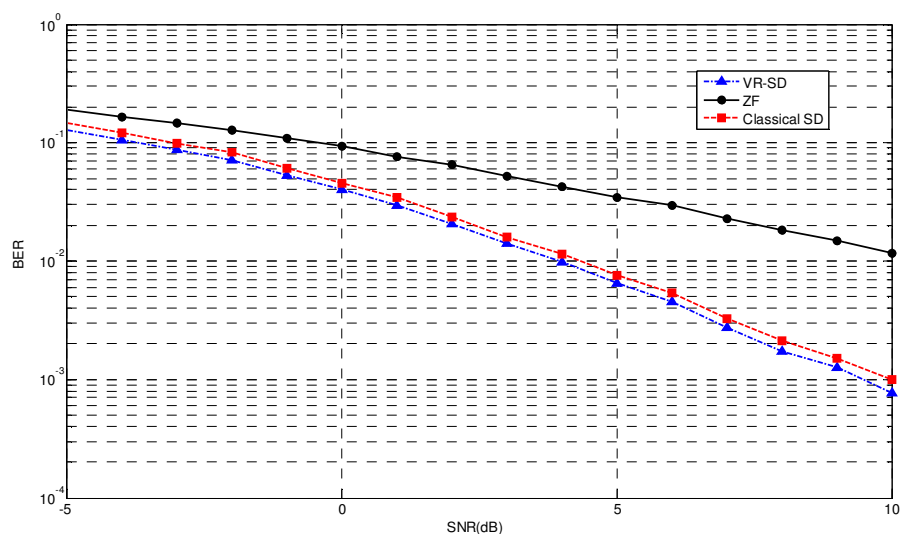

Figure 1. Comparison of BER between VR-SD, classical SD and ZF

Figure 1 shows the BER performance of VR-SD compared with classical SD algorithm and ZF algorithm. The computational complexity of ZF is much lighter than VR-SD algorithm, while the BER of VR-SD and classical SD is much better than ZF's. Because of the limitation of convergence times, the BER of classical SD algorithm doesn't have the same performance as the one which without limitation. Though the limitation of convergence times decreases the computational complexity, it still has some bad influence on its BER. Considering this, it is not strange that the BER of classical SD is not as well as the VR-SD's. We still cannot ignore the fact that if the times of convergence circulation keep rising, the BER of classical SD algorithm will be better than VR-SD.

\section{CONCLUSIONS}

This paper solve the problem of the algorithm complexity of convergence radius by designing a SD algorithm strategy-VR-SD algorithm. The VR-SD algorithm can avoid the unnecessary computational complexity caused by an unsuitable value of original convergence radius. Subjected to the same BER, both the classical SD and the VR-SD algorithm have a nearly $3 \mathrm{~dB}$ gain from the ZF algorithm. While the gain between the classical SD algorithm and the VR-SD algorithm is about $0.5 \mathrm{~dB}$. Considering the time overhead of classical SD algorithm, which convergence times is 5 , is $4 \%$ more than the VR-SD algorithm's. The gain of $0.5 \mathrm{~dB}$ from classical SD to VR-SD is a really great improvement. It demonstrates that the use of variable radius strategy raises the BER of SD algorithm in a considerable degree.

\section{ACKNOWLEDGEMENTS}

The paper is sponsored by "the Fundamental Research Funds for the Central University" (Grant No.HIT.NSRIF.2015024).

\section{REFERENCES}

[1] Vikalo H, Hassibi B \& Kailath T, (2004) "Iterative decoding for MIMO channels via modified sphere decoding", IEEE Transactions on Wireless Communications, Vol. 3, No. 6, pp2299-2311.

[2] Lampe L, Schober R \& Pauli V, (2004) "Multiple-symbol differential sphere decoding", IEEE Transactions on Communications, Vol. 53, No. 12, pp1981-1985.

[3] Ding Y, Wang Y R \& Li N, (2014) "Widely linear sphere decoding by exploiting the hidden properties of PSK signals", IEEE Globecom 2014- Signal Processing for Communications Symposium (GC14 SPC). 
[4] Soltani. M.D., Alimadadi. M \& Amindavar, H, (2014) "A hybrid method to reduce the complexity of k-best sphere decoding algorithm”, 2014 22nd Iranian Conference on Electrical Engineering (ICEE), pp1765-1770.

[5] El-Khamy. M, Vikalo. H., Hassibi, B \& McEliece, R.J, (2009) "Performance of sphere decoding of block codes", IEEE Transactions on Communications, Vol. 57, No. 10, pp2940-2950.

[6] Viet-Hoa Nguyen, Berder. O. \& Scalart, P, (2013) "On the efficiency of sphere decoding for linearly precoded MIMO systems", Wireless Communications and Networking Conference (WCNC), 2013 IEEE, pp4021-4025.

[7] Li Shi-ping, Wang Long \& Chen Fang-chao, (2012) "Ordered sphere decoding detection algorithm for MIMO systems”, 2012 24th Chinese Control and Decision Conference (CCDC), pp3322-3325.

[8] Xu Ren \& Huahua Wang, (2012) "Research of Sphere Decoding Detection Algorithm in LTE-A System", 2012 8th International Conference on Wireless Communications, Networking and Mobile Computing (WiCOM), pp1-4.

[9] Singh. A, Elia. P \& Jalden. J, (2013) "Rate-reliability-complexity tradeoff for ML and lattice decoding of full-rate codes", 2013 IEEE International Symposium on Information Theory Proceedings (ISIT), pp1267-1271.

\section{AUTHORS}

Wu Di was born in 1990, and get his B.S degree in Harbin Institute of Technology in 2013.He is now getting his M.S degree in the same school. His studying field is about the wireless communication and the signal detecting of MIMO.

Li Dezhi was born in 1981. He has received his $\mathrm{PhD}$ degree in Harbin Institute of Technology. He is the lecturer in school of electronics and information Engineering. His main research fields are satellite communication, wireless multimedia transmission and wireless communication.

Wang Zhenyong received his B.S. and M.S. degrees in communication and information system from Harbin Institute of Technology in 2000 and 2002, respectively. He is the lecturer in school of electronics and information Engineering. His main research interests are cross-layer design, satellite communication, wireless multimedia transmission, and wireless heterogeneous network
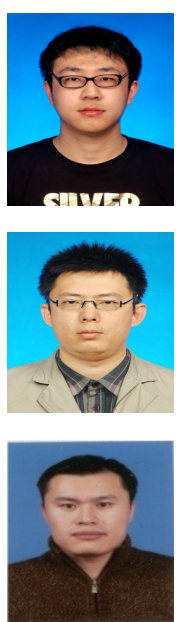